\title{
Archéopages
}

Archéopages

Archéologie et société

Hors-série 1 | 2008

Constructions de l'archéologie

\section{Trente ans après Leroi-Gourhan, où en sont les paléolithiciens?}

\section{Françoise Audouze}

\section{(2) OpenEdition}

1 Journals

Édition électronique

URL : https://journals.openedition.org/archeopages/831

DOI : 10.4000/archeopages.831

ISSN : 2269-9872

Éditeur

INRAP - Institut national de recherches archéologiques préventives

Édition imprimée

Date de publication : 1 février 2008

Pagination : $7-11$

ISSN : 1622-8545

\section{Référence électronique}

Françoise Audouze, "Trente ans après Leroi-Gourhan, où en sont les paléolithiciens ? ", Archéopages

[En ligne], Hors-série 1 | 2008, mis en ligne le 01 février 2008, consulté le 24 février 2023. URL : http:// journals.openedition.org/archeopages/831 ; DOI : https://doi.org/10.4000/archeopages.831

Tous droits réservés 


\section{Trente ans après \\ Leroi-Gourhan, où en sont les paléolithiciens?}

Françoise Audouze

CNRS, UMR 7041 "Archéologies et Sciences de l'Antiquité»

D ans un article de 2004, Jean-Paul Demoule a mis en lumière avec finesse certains des apports majeurs de l'œuvre de LeroiGourhan, en même temps que les conséquences négatives de son choix stratégique d'une archéologie de terrain très sélective. Il soulignait que son parti pris en faveur de la fouille raffinée de quelques sites exceptionnels, au détriment du sauvetage du plus grand nombre, l'avait conduit à négliger le développement de l'archéologie préventive, très en retard en France par rapport à la plupart des pays européens. Vingt ans après sa mort, il nous paraît intéressant d'observer l'évolution des recherches en archéologie du Paléolithique, dans les domaines que Leroi-Gourhan avait marqués de ses idées et de ses interventions en matière de politique scientifique. $^{\mathbf{1}}$

Entre continuité et rupture. D'un côté, l'aprèsLeroi-Gourhan se caractérise par la continuité, l'approfondissement des méthodes et l'expansion des problématiques qui ont abouti à une bien meilleure perception des sociétés du Paléolithique supérieur. De l'autre, de profondes ruptures sont intervenues dans l'étude des hommes du Pléistocène moyen, tant dans la perception de leurs capacités cognitives que par les recherches sur l'origine des comportements modernes et sur l'ADN des hommes fossiles. Enfin, même s'il demeure une façon française marquée par la palethnologie de faire des recherches en Préhistoire, les paléolithiciens français sont partie prenante de l'évolution internationale de la recherche, tout en maintenant une certaine distance par rapport aux grands débats théoriques anglo-saxons.

Continuités et innovations. Il n'y a pas aujourd'hui de succession à l'audacieuse entreprise d'une science unifiée de l'homme. Éric Boëda s'inscrit toutefois dans la lignée technologique de Leroi-Gourhan en construisant une théorie sur la genèse des industries lithiques qui s'appuie sur les acquis de la chaîne opératoire, la théorie du «mode d'existence des objets techniques» de Simondon et les concepts ergonomiques de la relation entre le geste et l'instrument de Pierre Rabardel (Boëda 2005).

Depuis vingt-cinq ans, les recherches ont considérablement avancé notre connaissance des sociétés paléolithiques grâce aux travaux sur la technologie sans qu'un changement de paradigme se soit révélé nécessaire. On peut l'expliquer par l'adoption généralisée du concept et de la méthode analytique de la chaîne opératoire et la synthèse opérée entre ces derniers et les concepts et méthodes d'analyse développés par l'école de Jacques Tixier sur la base des connaissances pratiques de François Bordes. Le développement puis l'adoption des notions d'intentionnalité, de savoir-faire, de productivité et de niveaux de compétence dans la taille du silex ont conduit tous les paléolithiciens français à utiliser des standards d'analyse communs. Le développement des concepts d'économie de la matière première et d'économie du débitage a permis l'essor des problématiques économiques fondées sur les matières premières. On est passé de la description des cultures à la compréhension de la logique de systèmes techniques intégrés dans des systèmes techno-économiques. On ne parle plus de cultures mais plus modestement de «techno-faciès». Appliquée d'abord au lithique puis aux autres matériaux, l'analyse des microtraces d'utilisation a fait passer l'étude des outils du descriptif à l'analyse fonctionnelle et à l'identification des tâches auxquelles ils sont associés. L'adoption de standards de fouilles élevés a permis des questionnements plus poussés sur l'organisation et la fonction des habitats, la saisonnalité et la division sexuelle du travail, notamment dans les sites magdaléniens et aziliens du Bassin parisien.

Un autre facteur de progrès commun à l'ensemble de l'archéologie a été l'élargissement constant des champs d'investigation, la mise en œuvre de la pluridisciplinarité et une mise en perspective archéologique des disciplines associées: les termes de géoarchéologie et d'archéozoologie témoignent de ce développement intégré. En gestation dans les travaux de terrain de Leroi-Gourhan, l'accent mis sur l'environnement est devenu l'une des composantes principales des problématiques paléolithiques. La reconstitution du paysage et la prise en compte de l'éthologie des animaux chassés créent une dialectique «homme-milieu» qui a considérablement enrichi les interprétations. Cet intérêt va de pair avec l'élargissement des perspectives. Dans un certain nombre de cas privilégiés, c'est la région et non plus le site qui est prise comme échelle d'analyse pertinente, comme en témoigne l'utilisation de systèmes d'information géographiques.

Des modèles ethnoarchéologiques aux modèles a posteriori. Un apport décisif est venu de l'archéologie américaine ou plus exactement de l'un de ses plus illustres représentants, Lewis Binford, dont les modèles ethnoarchéologiques ont été largement adoptés, que ce soit l'index d'utilité nutritionnelle des os d'herbivores, l'organisation des rejets autour du foyer en drop et toss zones, les caractéristiques de composition faunistique des campements en fonction de leur usage ou les caractéristiques de la mobilité saisonnière des chasseurs-collecteurs (1983). À partir de ces propositions, un certain nombre de modèles a posteriori, fruits de va-et-vient entre des modèles théoriques et les données de fouille, 


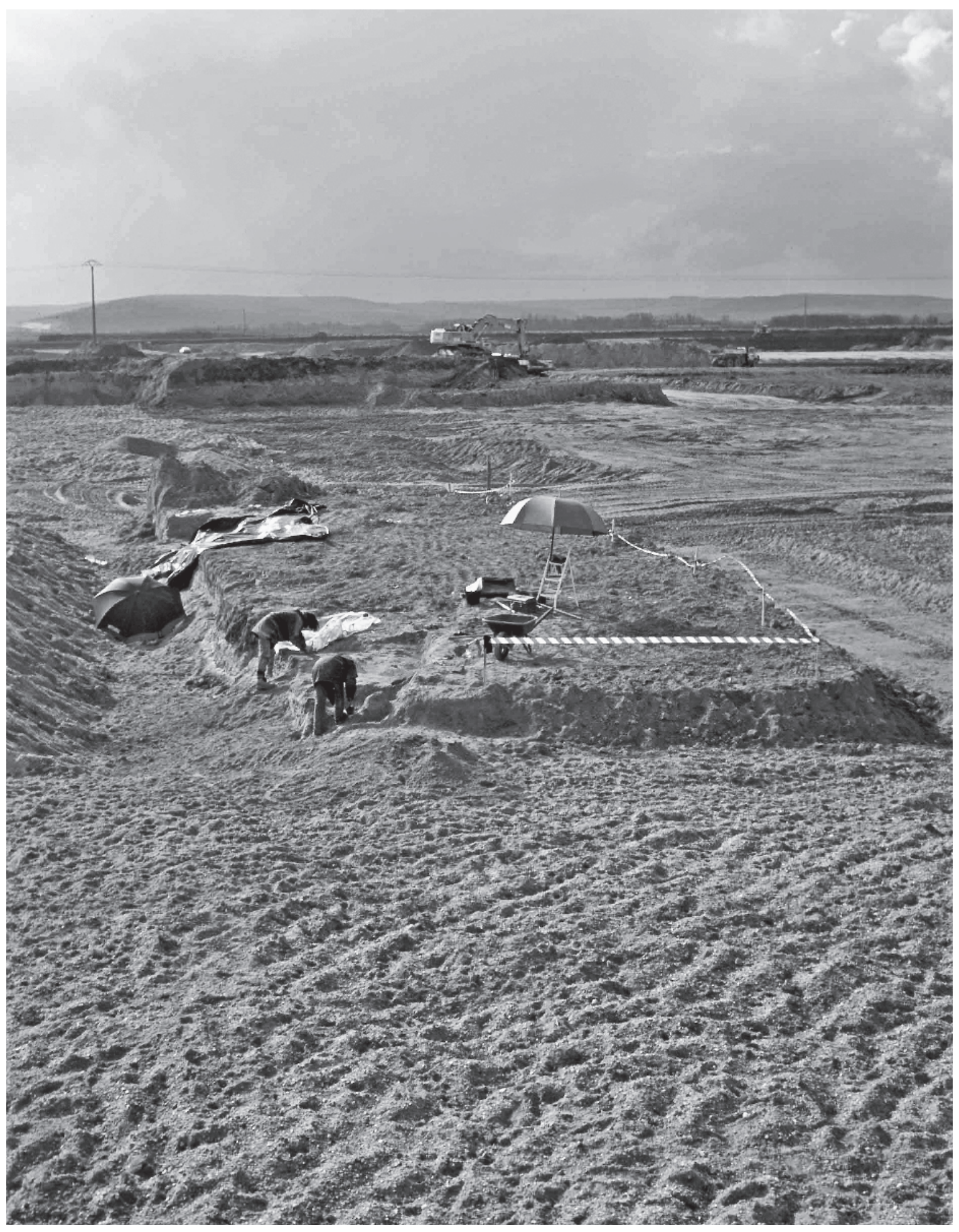

[Fig. 1] Le suivi archéologique

d'une gravière à Soucy, dans l'Yonne,

a conduit à la découverte

de six gisements du Pléistocène

moyen où la faune était conservée.

La fouille a apporté des informations

novatrices sur l'occupation

des vallées au Paléolithique ancien

et moyen, sur les modalités

de la chasse à ces époques reculées

et sur la variabilité des industries. 
ont vu le jour pour le Paléolithique supérieur; notamment des modèles saisonniers d'habitations et d'unités domestiques à partir des fouilles des sites magdaléniens du Bassin parisien, des modèles de mobilité saisonnière dans le Massif central et dans le centre du Bassin parisien. Ce n'est que récemment que des recherches ethnoarchéologiques se sont développées en milieu périglaciaire pour documenter le Paléolithique, notamment les projets «Ethnorenne» et «Système Renne», tandis que des études actualistes de taphonomie sont pratiquées depuis plus longtemps par des archéozoologues sur des terrains africains.

Les axes de rupture. Les recherches sur le Pléistocène moyen et les Néandertaliens sont depuis longtemps en rupture avec les idées de Leroi-Gourhan. Dans le débat enrichi par les nombreuses datations, les fouilles récentes et le réexamen des fouilles anciennes, les interstratifications de couches chatelperroniennes et aurignaciennes ont été éliminées, rendant aux Néandertaliens les innovations chatelperroniennes qui leur appartenaient. L'existence des sépultures néandertaliennes a été définitivement reconnue, tout comme l'existence de parures et d'objets manufacturés en os dans leur mobilier le plus récent (grotte du Renne d'Arcy-sur-Cure). Les Néandertaliens ont été placés à côté de notre lignée et le débat porte désormais sur leur interfécondité éventuelle avec les hommes anatomiquement modernes et sur les raisons de leur extinction. Le modèle multifocal proposé par Francesco d'Errico, João Zilhao et al. (2003) constitue un sérieux changement paradigmatique qui place l'apparition du symbolisme et du langage avant la transition entre Paléolithique moyen et Paléolithique supérieur. La modernisation de la culture serait intervenue de façon parallèle et indépendante, tantôt par complexification graduelle, tantôt par l'apparition soudaine de comportements nouveaux, d'abord chez les hommes modernes en Afrique et plus tardivement mais de façon indépendante chez les Néandertaliens européens.

L'art pariétal. On ne peut guère se satisfaire des progrès réalisés depuis trente ans dans le domaine de l'art, bien que la plupart des spécialistes d'art pariétal se soient déclarés en rupture avec les théories de Leroi-Gourhan. Seul Georges Sauvet (2004) se situe dans la continuité et montre la validité des associations préférentielles d'animaux sur les parois trouvées par LeroiGourhan, mais uniquement pour le Magdalénien, et avec le cheval comme seule espèce dominante. Le nombre des grottes ornées découvertes s'est accru. Elles ont fait l'objet de descriptions beaucoup plus précises à l'aide de moyens techniques puissants. Pourtant, l'élément marquant de la période n'est en rien théorique ni conceptuel. Il tient aux nombreuses datations ${ }^{14} \mathrm{C}$ effectuées, qui ont fait remonter l'art à l'Aurignacien et déplacé son origine vers l'est avec les grottes Chauvet, Cosquer et la Grande grotte d'Arcy. Quant à la théorie du shamanisme, prônée par David Lewis-Williams et Jean Clottes (2003), enfermée dans une explication générique qui fait - aux dires des ethnologues et des neurologues - la part trop belle à la transe, elle ne permet d'expliquer ni les variantes régionales ni la signification des représentations. L'amorce d'un renouveau se fait toutefois jour avec l'étude novatrice de Norbert Aujoulat, qui met en évidence un cycle de représentations à composantes saisonnières dans la grotte de Lascaux (2004).

L'erreur stratégique de Leroi-Gourhan enfin réparée? L'absence de Leroi-Gourhan (et d'autres grands paléolithiciens) dans la bataille pour l'archéologie préventive n'a pas permis à cette dernière de se développer aussi tôt qu'il l'aurait fallu. Une fois surmonté cette erreur stratégique, le retard est resté plus grand pour les périodes paléolithique et mésolithique. Les recrutements de la première génération d'archéologues à l'Afan puis à l'Inrap se sont faits majoritairement en faveur des périodes gallo-romaine et protohistorique, dont les sites étaient plus nombreux et plus visibles ou faciles à repérer en diagnostic. Si la Préhistoire ancienne a peu à peu gagné droit de cité en archéologie préventive, elle le doit à la création d'équipes de terrain où ont coopéré des paléolithiciens, des géologues et des géomorphologues dont les études microrégionales et locales ont permis d'identifier les zones ayant conservé les couches géologiques susceptibles de contenir des niveaux paléolithiques. L'aventure de l'autoroute A 5 et les découvertes de la vallée de la Vanne marquent à cet égard un tournant dans la sensibilisation des responsables de l'archéologie préventive. La découverte des sites du Paléolithique inférieur de Soucy dans l'Yonne [Fig.1], de Beauvais dans l'Oise ou du tracé de contournement de Bergerac représente autant d'illustrations du rôle essentiel des géoarchéologues aux côtés des paléolithiciens (Bourguignon, Lhomme 2004). L'archéologie préventive a, plus que toute autre, contribué à renouveler nos connaissances et notre compréhension de l'occupation du territoire par les hommes du Paléolithique ancien et moyen. C'est, entre autres accomplissements, une opération préventive qui a mis au jour la première organisation spatiale indéniable d'un campement de chasseurs moustériens.

Les paléolithiciens peuvent être reconnaissants à Jean-Paul Demoule et à l'Inrap d'avoir su convaincre les autorités de tutelle de l'intérêt d'une pratique pluridisciplinaire de l'archéologie préventive et du maintien de liens étroits avec la recherche. Ils sont parmi les premiers à en bénéficier. La constitution d'équipes pluridisciplinaires, les relations entretenues avec les milieux de la recherche assurent la fluidité de l'information d'un milieu à l'autre et l'adoption 


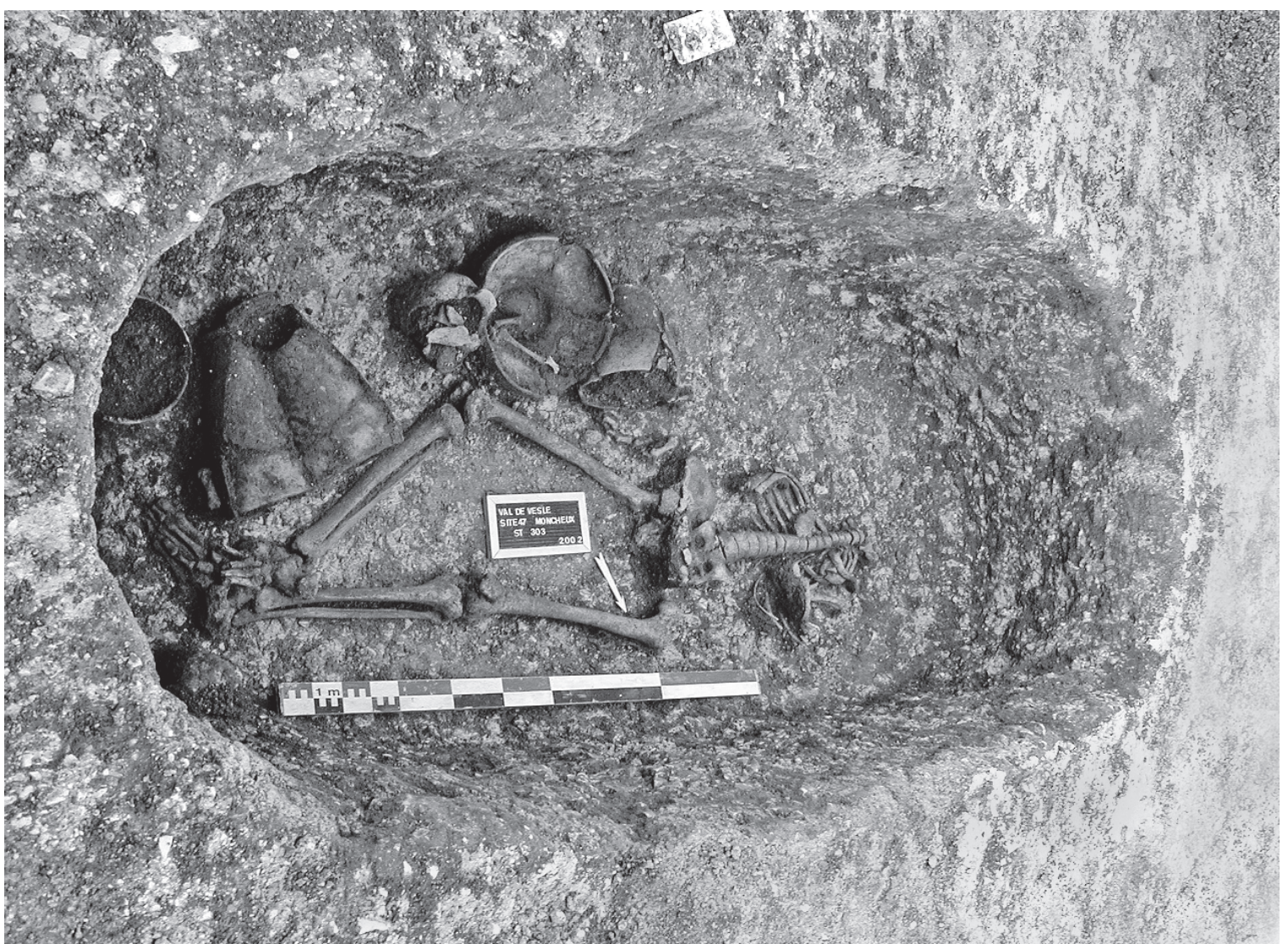

[Fig.1] Inhumation masculine

à Val de Vesle "Moncheux» (Marne)

Cette sépulture a été anciennement

sondée selon un procédé

caractéristique, appelé la sonde

champenoise: après avoir délimité

les contours de la fosse, un des angles

est de la tombe est vidé, le plus

souvent la céramique et l'essentiel

du squelette sont ainsi préservés. 
réciproque des innovations conceptuelles, méthodologiques et techniques.

Aujoulat N. 2004: Lascaux. Le geste, l'espace et le temps, Paris, Le Seuil. BINFORD L. 1983: In Pursuit of the Past, Decoding the Archaeological Record, New York, Thames \& Hudson.

BoËDA E. 2005: «Paléo-technologie ou anthropologie des Techniques?», Arob@se, www.arobase.to, vol.1, p.46-64.

BourguignON L., LHOMME V. 2004: «Les temps des chasseurscueilleurs. Le Paléolithique et le Mésolithique», in J.-P.Demoule, La France archéologique. Vingt ans d'aménagements et de découvertes, Paris, Hazan, p.36-47.

Clottes J. 2003: «De <l'art pour l'art' au chamanisme: l'interprétation de l'art préhistorique ", La Revue pour l'histoire du CNRS, nº 8 , mai 2003.

Demoule J.-P. 2004: «André Leroi-Gourhan, l'ethnie, la culture et le préhistorien: histoire d'un rendez-vous manqué», in F.Audouze, N.Schlanger (dir.), Autour de l'Homme. Contexte et actualité de Leroi-Gourhan, Antibes, Éditions APDCA, p. 45-67.

D'errico F., HenshilwoodC., Lawson G., VanhaerenM., TillieA.-M., Soressim., BressonF., MaureilleB., Nowella., LAKARraJ., BACKWELLL., JulienM. 2003: «Archaeological Evidence for the Emergence of Language, Symbolism and Music: an Alternative Multidisciplinary Perspective», Journal of World Prehistory, p.1-7o.

SAUVET G. 2004: «Langage préhistorique, langages de préhistoriens", in F.Audouze, N.Schlanger (dir.), Autour de l'Homme. Contexte et actualité de Leroi-Gourhan, Antibes, Éditions APDCA, p.249-270.

\section{Archéologie funéraire de l'âge du Fer en Champagne-Ardenne}

Lola Bonnabe

Inrap, UMR 7041 "Archéologies et Sciences de l'Antiquité»

D ans sa préface à Chronologie et société dans les nécropoles celtiques de la culture Aisne-Marne de Jean-Paul Demoule (1999), Christian Goudineau écrit: «rares furent les naufrages aussi complets que celui qui a englouti ces dizaines de milliers de tombes champenoises et l'essentiel de leur contenu: ont sombré corps et biens entre quatre et cinq cents nécropoles». La région de ChampagneArdenne a en effet été fortement occupée pendant le deuxième âge du Fer et est probablement la plus riche en sépultures de La Tène. Reste qu'il est peu d'autres régions où le passé a été aussi sauvagement ravagé.

Les travaux qui ont précédé la publication évoquée ci-dessus sont fondateurs et servent aujourd'hui de socle aux recherches sur les nécropoles de lâge du Fer en Champagne et dans la vallée de l'Aisne. Une typo-chronologie des objets a été mise en place, une culture matérielle, l'«Aisne-Marne», a été définie et une caractérisation des pratiques funéraires et de leurs variations chronologiques et régionales a été proposée. L'organisation de la société, en particulier pour ce qui est de la hiérarchie, et la relation homme-femme ont également été abordées.

Depuis les années 1990, en ChampagneArdenne, une équipe d'archéologues du funéraire gère, dans le cadre préventif, la quasi-totalité des fouilles sépulcrales, toutes périodes confondues, selon une procédure dite «protocole champenois». Parallèlement, une équipe pluridisciplinaire s'est constituée pour étudier les vestiges funéraires de l'âge du Fer. ${ }^{\mathbf{1}}$ Aujourd'hui, la diversité des types d'analyse s'accompagne d'une multiplicité de points de vue sur les vestiges, tandis que les problématiques sociétales sont de plus en plus variées.

Ce texte ne vise pas à retracer en détail l'histoire de l'archéologie funéraire de lâge du Fer en Champagne-Ardenne, mais plutôt à en évoquer les phases principales.

\section{Le $\mathrm{XIX}^{\mathrm{e}}$ siècle, l'eldorado champenois.}

Ce pourtour oriental du Bassin parisien est un gisement exceptionnel en sépultures de l'âge du Fer. Dès la fin du XviII ${ }^{e}$ siècle et surtout pendant la seconde moitié du XIX', grâce à l'impulsion donnée par Napoléon III, les sépultures sont exploitées comme de véritables mines d'objets qui, outre leur beauté intrinsèque, permettent d'alimenter une construction identitaire nationale gauloise en opposition à celle, germanique, des royaumes allemands qui, unis derrière le royaume de Prusse, ont vaincu la France en 1870. Depuis lors, la confusion entre Gaule et France n'aura cessé de nourrir le mythe d'une France millénaire, peu soucieuse de la réalité historique.

Tandis que des scientifiques notables s'intéressent à l'Antiquité classique, les acteurs des pillages ${ }^{2}$ des tombes champenoises - agissant pour le compte de plus ou moins riches commanditaires ou de leurs intermédiaires développent des techniques pour mettre en évidence les fosses sépulcrales. Leur tâche est considérablement facilitée par le contexte sédimentaire régional: les tombes sont des fosses creusées dans de la craie à la fois poreuse et dure et remplies d'une terre brune et meuble. Il suffit aux chercheurs de trésors d'observer la végétation, plus sombre et plus fournie à l'emplacement des tombes, voire d'utiliser la résonance des sabots d'un cheval. ${ }^{3}$ La plus célèbre de ces techniques - la sonde champenoise - est sans doute à l'origine des ravages les plus systématiques survenus au cours de la seconde moitié du XIX ${ }^{\mathrm{e}}$ siècle. ${ }^{4}$ Cet instrument permet de délimiter les contours des fosses sépulcrales par enfoncements successifs dans la terre végétale. Certains vivent de la vente des objets, d'autres constituent des collections ${ }^{5}$ parfois accompagnées de planches illustratives somptueuses, dont l'objectif est davantage la présentation d'objets prestigieux qu'un véritable inventaire ou qu'un classement des découvertes. Ailleurs, pourtant, les objets archéologiques sont intégrés dans des classifications construites sur les modèles naturalistes élaborés dès le XVIII ${ }^{e}$ siècle et durant le XIX ${ }^{e}$. Entre la fin du XIX ${ }^{e}$ et le début du XX $x^{e}$ siècle, les squelettes exhumés alimentent, quant à eux, les recherches des laboratoires parisiens d'anthropologie physique.

Les inventaires estimatifs des tombes sont impressionnants: trois mille cinq cents tombes en 1876, d'après Alexandre Bertrand (1820-1902), huit mille en 1881, d'après Auguste Nicaise (1828-1900) (Demoule 1999, p.15). 